\title{
Fatores associados à continuidade do aleitamento materno por 12 meses ou mais em mulheres trabalhadoras de um hospital geral
}

\author{
Factors associated with breastfeeding continuation for 12 months \\ or more among working mothers in a general hospital
}

Michele Saraiva Mendes (https://orcid.org/0000-0002-3240-4339) ${ }^{1}$

Monique Schorn (https://orcid.org/0000-0002-0258-5052) ${ }^{1}$

Lilian Cordova do Espírito Santo (https://orcid.org/0000-0002-2577-9079) ${ }^{2}$

Luciana Dias Oliveira (https://orcid.org/0000-0002-3438-0668) ${ }^{3}$

Elsa Regina Justo Giugliani (https://orcid.org/0000-0001-6569-6473) ${ }^{1}$
${ }^{1}$ Programa de PósGraduação em Saúde da

Criança e do Adolescente, Universidade Federal do Rio Grande do Sul (UFRGS). R. Ramiro Barcelos 2400, Santa Cecília. 90035-003 Porto Alegre RS Brasil. michele.mendes@ yahoo.com.br

${ }^{2}$ Departamento de Enfermagem Materno Infantil, UFRGS. Porto Alegre RS Brasil.

${ }^{3}$ Departamento de Nutrição, UFRGS. Porto Alegre RS Brasil.

\begin{abstract}
This article aims to identify factors associated with breastfeeding continuation for at least 12 months among working mothers in a hospital in the south of Brazil. We conducted a cross-sectional study, interviewing 251 women who breastfed after returning to work. Eligibility criteria included non-twin biological children aged between 12 and 36 months and the absence of an illness (mother and/or child) that could affect breastfeeding. The association between breastfeeding continuation and the exposure variables was tested using Poisson multivariate regression. Only one work-related variable showed a significant association with the outcome. Working only during the day increased the prevalence of BF continuation for at least 12 months by 37\%. The following non-work-related factors showed a positive association with the outcome: mothers without a college degree; mothers with at least 12 months' prior breastfeeding experience; child not given milks other than breast milk when the mother returned to work, and not using a pacifier. The following variables showed a negative association with the outcome: older maternal age; older gestational age; mother receiving support from the child's caregiver; and mother receiving professional breastfeeding support. Non-work-related factors had a greater influence on breastfeeding continuation for at least 12 months among working mothers.
\end{abstract}

Key words Breastfeeding, Working mothers, Risk factors
Resumo O objetivo deste estudo é identificar fatores associados à continuidade da amamentação por 12 meses ou mais em mulheres trabalhadoras. Estudo transversal realizado por meio de entrevista com 251 trabalhadoras de um hospital, com filhos biológicos entre 12 e 36 meses de idade, não gemelares e sem doença que afetasse a amamentação, e amamentando quando do seu retorno ao trabalho. Para a associação entre a continuidade da amamentação e as variáveis de exposição utilizou-se a regressão multivariável de Poisson. Apenas uma variável relacionada ao local de trabalho da mulher mostrou associação significativa com o desfecho. Trabalhar durante o dia aumentou em $37 \%$ a prevalência da amamentação por 12 meses ou mais. Os fatores não relacionados ao trabalho da mulher que mostraram associação positiva com o desfecho: mãe sem curso superior, experiência de amamentação superior a 12 meses; criança não receber outro leite quando a mãe retornou ao trabalho e não ter usado chupeta. Por outro lado, maior idade da mãe, maior idade gestacional, apoio do cuidador da criança e apoio profissional na amamentação associaram-se negativamente ao desfecho. Fatores não relacionados diretamente ao trabalho materno tiveram maior participação na continuidade da amamentação por 12 meses ou mais.

Palavras-chave Aleitamento materno, Trabalho feminino, Fatores de risco 


\section{Introdução}

Tendo em vista o impacto positivo do aleitamento materno (AM) na saúde da criança e da mulher que amamenta ${ }^{1-3}$, a prevenção do desmame precoce impõe-se, mesmo quando o desejo da mulher é o de manter a amamentação por mais tempo.

Entre as múltiplas razões para a interrupção precoce do AM, sobretudo do aleitamento materno exclusivo (AME), está o trabalho materno ${ }^{4-6}$. Entretanto, há estudos mostrando que medidas de apoio à manutenção da amamentação após o retorno da mulher ao trabalho têm impacto positivo na continuidade do AM. Entre essas medidas destacam-se políticas de flexibilização de horários, pausas para amamentar ou extrair o leite materno (LM), local apropriado para retirada e armazenamento do leite, apoio do empregador e dos colegas de trabalho ${ }^{7}$, e disponibilidade de creches no local ou próximo ao trabalho ${ }^{6}$.

Pesquisa de âmbito nacional ${ }^{8}$ mostrou que 26,8\% das mulheres com filhos menores de 6 meses trabalhando fora estavam amamentando exclusivamente, enquanto que $53,4 \%$ daquelas em licença-maternidade e $43,9 \%$ das que não trabalhavam fora mantinham esse padrão de amamentação. Entre as mulheres com crianças menores de 1 ano, $81,2 \%$ das que não trabalhavam fora estavam amamentando, contra $65 \%$ das que trabalhavam fora, evidenciando, assim, o impacto do trabalho materno nas taxas de AM.

$\mathrm{O}$ apoio à amamentação no local de trabalho é citado na literatura como um importante fator que influencia a continuidade do AM após o retorno da mulher ao trabalho. Apesar da importância do trabalho materno como fator de risco à manutenção do $\mathrm{AM}$, há poucos estudos publicados sobre o assunto, sobretudo no Brasil. Pouco se conhece acerca das dificuldades e facilidades relacionadas à continuidade do AM após o retorno das mulheres ao trabalho. A presente pesquisa foi idealizada considerando-se que o conhecimento de fatores que interferem na amamentação da mulher trabalhadora é importante para o planejamento de ações que visem à ampliação da duração do AM nessa população. Assim, o seu objetivo foi identificar fatores associados à continuidade do AM por 12 meses ou mais em mulheres trabalhadoras de um hospital geral de grande porte, com ênfase nos fatores relacionados ao local de trabalho.

\section{Materiais e métodos}

Este é um estudo transversal, com coleta de dados entre junho de 2016 e julho de 2017, realizado no município de Porto Alegre, sul do Brasil, com 251 servidoras de um hospital público geral de grande porte ligado a uma universidade.

O hospital possui cerca de 6.000 servidores, sendo a maioria do sexo feminino e em idade fértil. Oferece as seguintes facilidades para as servidoras com filhos pequenos: licença-maternidade de 180 dias, liberação de uma hora por dia para amamentação até a criança completar 1 ano de vida; creche localizada próximo à instituição, banco de leite humano (BLH), serviço de disque-amamentação e profissionais capacitados em AM que atuam na unidade de internação neonatal como consultores em lactação.

Participaram da pesquisa trabalhadoras do hospital que atendiam aos seguintes critérios de inclusão: ter filhos biológicos com idade entre 12 e 36 meses, não gemelares, nascidos durante o contrato de trabalho com a instituição; estar amamentando após a licença-maternidade; e não apresentarem doenças (mães e/ou crianças) que afetassem significativamente o AM, tais como malformações orofaciais e doenças neurológicas.

Com o intuito de obter a informação sobre o desfecho, definido como a continuidade do AM por 12 meses ou mais, foi estabelecida a faixa etária mínima de 12 meses. A idade máxima de 36 meses foi estipulada para minimizar um possível viés de memória.

Caso a mulher tivesse mais de um filho na faixa etária do estudo, foi selecionada a criança de menor idade.

As informações foram obtidas por meio de entrevista previamente agendada com as mulheres nas dependências do hospital, utilizando-se questionário estruturado elaborado pelas pesquisadoras especialmente para a pesquisa. As entrevistas tiveram duração média de 30 minutos.

Para o cálculo amostral, foram utilizados os seguintes parâmetros: alfa $=5 \%$; poder do estudo $=80 \%$; prevalência de AM por 12 meses ou mais $=50 \%$; prevalência das principais variáveis independentes segundo estudo piloto realizado com 7 mulheres $=50$ a $60 \%$; razão de chances (odds ratio - OR) =2,0. O cálculo resultou em uma amostra mínima variando de 296 a 317, dependendo das frequências das variáveis independentes.

Os dados coletados foram duplamente digitados com posterior validação e as análises estatísticas feitas pelo software SPSS versão 23. As variáveis quantitativas foram descritas por média 
e desvio padrão ou mediana e amplitude interquartílica; as categóricas por frequências absolutas e relativas.

Devido ao elevado número de variáveis envolvidas para explicar o desfecho, foi elaborado um modelo hierárquico de regressão ${ }^{10}$, em que as variáveis são agrupadas em blocos de acordo com a sua relação de proximidade com o desfecho ${ }^{11}$. Assim, as diferentes variáveis foram distribuídas em três níveis hierárquicos, a saber: distal, intermediário e proximal. A Figura 1 lista as variáveis que foram exploradas em cada nível hierárquico quanto a uma possível associação com a continuidade do AM por 12 meses ou mais na população estudada.

Primeiramente, estimou-se a associação entre o desfecho e as variáveis de cada bloco, utilizando-se a regressão de Poisson univariável. As vari- áveis que atingiram nível de significância $\mathrm{p}<0,20$ na análise univariável foram submetidas à regressão de Poisson multivariável, ou seja, submetidas juntas no seu bloco à regressão de Poisson (análise intrabloco). A variável que atingia nível de significância $\mathrm{p}<0,20$ na análise intrabloco permanecia no modelo até o final, ajustando os efeitos nos blocos subsequentes por serem considerados possíveis fatores de confusão. A magnitude da associação foi estimada por meio de razão de prevalência bruta (RP) e ajustada (RPa) e seus respectivos intervalos de confiança de 95\% (IC95\%), sendo considerada significativa a associação com valor de $\mathrm{p}<0,05$. A pesquisa foi aprovada pelo Comitê de Ética em Pesquisa do hospital que sediou a pesquisa e cadastrada na Plataforma Brasil. Todas as participantes assinaram o Termo de Consentimento Livre e Esclarecido (TCLE).

\section{NÍVEL DISTAL}

Idade da mãe, escolaridade, cor da pele, paridade, coabitação com companheiro, coabitação com a própria mãe, experiência prévia em amamentação.

NÍVEL INTERMEDIÁRIO
Local do acompanhamento pré-natal, intenção quanto à duração da amamentação, intenção de amamentar
após o retorno ao trabalho, local do parto, tipo de parto, idade gestacional, sexo da criaça, alta da
maternidade em aleitamento materno exclusivo, amamentação na primeira hora de vida.

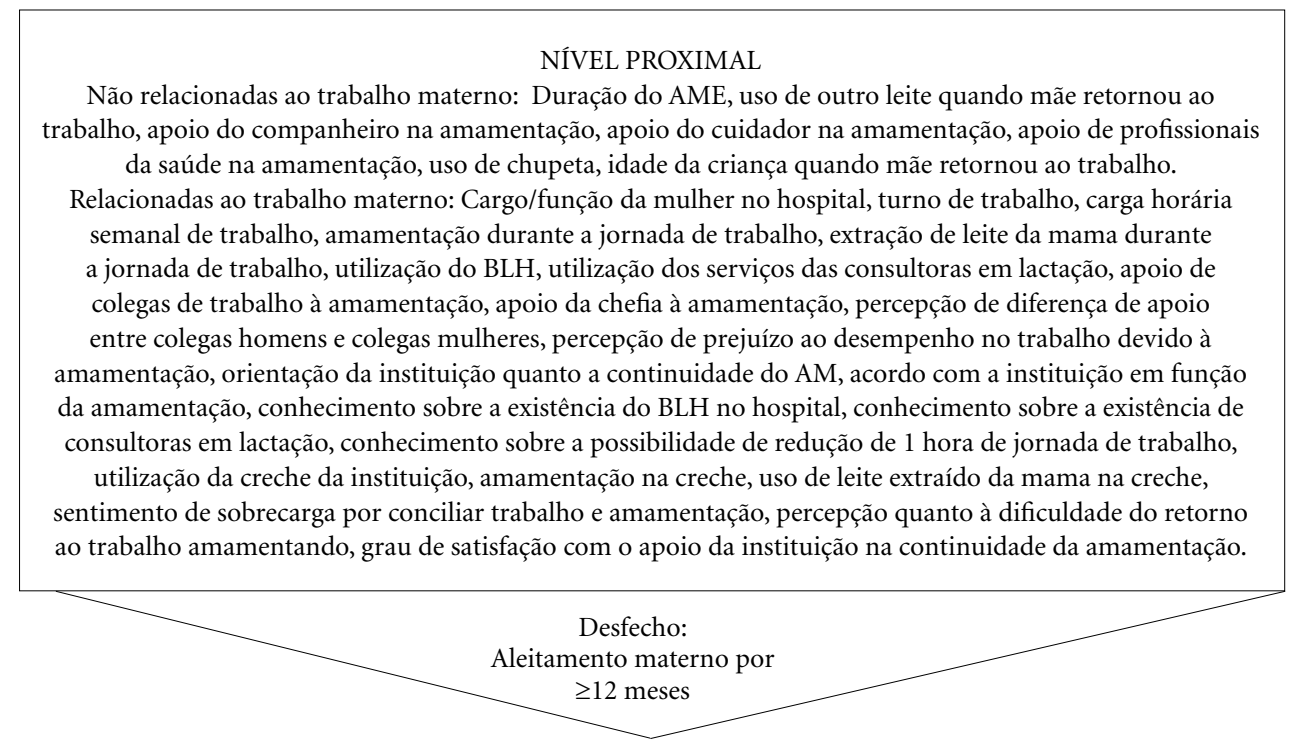

Figura 1. Modelo hierárquico conceitual utilizado para identificar os fatores associados com a continuidade do AM por $\geq 12$ meses em mulheres trabalhadoras. 


\section{Resultados}

Inicialmente, foram contatadas 300 mulheres. Dessas, 49 não foram incluídas no estudo por não estarem amamentando quando do retorno ao trabalho. Portanto, participaram deste estudo 251 mulheres que atendiam aos critérios de elegibilidade. A Tabela 1 apresenta as características da população estudada.

Tabela 1. Características sociodemográficas, antecedentes obstétricos, amamentação e trabalho da mulher na população estudada. Porto Alegre-RS, 20162017 $(n=251)$.

\begin{tabular}{|c|c|}
\hline Variáveis & n (\%) \\
\hline \multicolumn{2}{|l|}{ Sociodemográficas } \\
\hline Idade média $\_\mathrm{DP}$ (anos) & $35 \pm 3,7$ \\
\hline Curso superior completo & $149(59,4)$ \\
\hline Cor da pele da mãe branca & $213(84,9)$ \\
\hline Coabita com companheiro & $234(93,2)$ \\
\hline Coabita com a própria mãe & $9(3,6)$ \\
\hline Sexo da criança - feminino & $110(43,8)$ \\
\hline \multicolumn{2}{|l|}{ Antecedentes Obstétricos } \\
\hline Primíparas & $146(58,2)$ \\
\hline Acompanhamento pré-natal & $251(100,0)$ \\
\hline $\begin{array}{l}\text { Pré-natal na rede privada/ } \\
\text { conveniada de saúde }\end{array}$ & $238(94,8)$ \\
\hline $\begin{array}{l}\text { Parto no sistema conveniado/ } \\
\text { privado }\end{array}$ & $210(83,3)$ \\
\hline $\begin{array}{l}\text { Idade gestacional média } \pm \mathrm{DP} \\
\text { (semanas) }\end{array}$ & $38,2 \pm 1,7$ \\
\hline Parto cesáreo & $206(82,1)$ \\
\hline $\begin{array}{l}\text { Intenção quanto à duração } \\
\text { amamentação } \geq 12 \text { meses }\end{array}$ & $235(93,0)$ \\
\hline $\begin{array}{l}\text { Intenção de amamentar após o } \\
\text { retorno ao trabalho }\end{array}$ & $244(97,2)$ \\
\hline \multicolumn{2}{|l|}{ Amamentação } \\
\hline $\begin{array}{l}\text { Amamentação na primeira hora } \\
\text { de vida }\end{array}$ & $150(59,8)$ \\
\hline $\begin{array}{l}\text { Experiência prévia com } \\
\text { amamentação } \geq 12 \text { meses }\end{array}$ & $46(18,3)$ \\
\hline $\begin{array}{l}\text { Amamentação da criança } \geq 12 \\
\text { meses }\end{array}$ & $156(62,3)$ \\
\hline $\begin{array}{l}\text { Amamentação exclusiva na alta da } \\
\text { maternidade }\end{array}$ & $215(85,7)$ \\
\hline $\begin{array}{l}\text { Recebeu apoio do companheiro na } \\
\text { amamentação }\end{array}$ & $232(92,4)$ \\
\hline $\begin{array}{l}\text { Recebeu apoio do cuidador na } \\
\text { amamentação }\end{array}$ & $203(80,9)$ \\
\hline $\begin{array}{l}\text { Recebeu apoio profissional na } \\
\text { amamentação }\end{array}$ & $177(70,5)$ \\
\hline $\begin{array}{l}\text { Criança recebia outro leite quando } \\
\text { mãe retornou ao trabalho }\end{array}$ & $238(94,8)$ \\
\hline Uso de chupeta & $161(64,1)$ \\
\hline
\end{tabular}

A duração mediana do AME foi de 150 dias e 95\% das crianças estavam recebendo outro tipo de leite na época do retorno da mulher ao trabalho, o que ocorreu entre 4 e 11 meses, com mediana de 6 meses.

A maioria das mulheres relatou que pretendia amamentar por mais de 12 meses e praticamente todas disseram que gostariam de continuar a amamentação após o retorno ao trabalho; $62,3 \%$ das crianças foram efetivamente amamentadas por 12 meses ou mais.

A Tabela 2 mostra alguns dados relacionados com o retorno ao trabalho na condição de lactante. A maioria das mulheres trabalhava na assistência aos pacientes e apenas durante o dia. A média da carga horária semanal foi de 35,5 horas. Aproximadamente $3 / 4$ das mulheres relataram não ter sido informadas pela instituição a respeito de assuntos ligados à amamentação após o retorno ao trabalho, e o nível de desconhecimento sobre algumas facilidades da instituição variou de acordo com a facilidade.

Em torno de 1/3 das servidoras extraiu leite da mama durante a jornada de trabalho e/ou procuraram o BLH. Pouco menos de $1 / 4$ das crianças frequentou a creche e $13,5 \%$ foram amamentadas nesse local. Quando questionadas sobre o espaço destinado à amamentação na creche, mais da metade das mulheres que amamentaram nesse local o considerou inadequado.

A Tabela 3 apresenta o modelo final da análise multivariável hierarquizada estimando as associações entre a continuidade da amamentação por 12 meses ou mais e as diversas variáveis exploradas.

O resultado final da análise mostrou que os seguintes fatores apresentaram associação positiva com a continuidade da amamentação por 12 meses ou mais: mães sem curso superior; mães com experiência prévia de amamentação igual ou maior que 12 meses; criança não estar recebendo outro leite quando do retorno da mãe ao trabalho; criança não ter usado chupeta; e mãe trabalhar somente durante o dia. Por outro lado, os seguintes fatores mostraram associação negativa com o desfecho: idade da mãe (prevalência $3 \%$ menor a cada ano de idade); idade gestacional do recém-nascido (prevalência 7\% menor para cada semana a mais de gestação); mãe ter apoio do cuidador da criança e apoio profissional na amamentação. 
Tabela 2. Dados relacionados ao trabalho da mulher e amamentação. Porto Alegre-RS, 2016-2017 (n=251).

\begin{tabular}{|c|c|c|}
\hline Variáveis & $\mathbf{n}$ & $\%$ \\
\hline \multicolumn{3}{|l|}{ Cargo/função da mãe } \\
\hline Assistencial & 198 & 78,9 \\
\hline Apoio & 19 & 7,6 \\
\hline Administrativo & 34 & 13,5 \\
\hline \multicolumn{3}{|l|}{ Turno de trabalho da mãe } \\
\hline Diurno & 186 & 74,1 \\
\hline Noturno/Ambos & 65 & 25,9 \\
\hline Carga horária semanal de trabalho (horas) ${ }^{\star}-$ Média (DP) & $35,5(5,3)$ & \\
\hline \multicolumn{3}{|l|}{ Amamentação durante a jornada de trabalho } \\
\hline Sim & 56 & 22,3 \\
\hline Não & 195 & 77,7 \\
\hline \multicolumn{3}{|l|}{ Utilização dos serviços do banco de leite humano } \\
\hline Sim & 86 & 34,3 \\
\hline Não & 165 & 65,7 \\
\hline \multicolumn{3}{|l|}{ Utilização dos serviços das consultoras em lactação } \\
\hline $\operatorname{Sim}$ & 25 & 10,0 \\
\hline Não & 226 & 90,0 \\
\hline \multicolumn{3}{|l|}{ Apoio de colegas de trabalho à amamentação } \\
\hline Sim & 150 & 59,8 \\
\hline Não & 101 & 40,2 \\
\hline \multicolumn{3}{|c|}{ Percepção de diferença de apoio entre colegas homens e mulheres } \\
\hline Mulheres apoiam mais & 49 & 19,5 \\
\hline Homens apoiam mais/sem diferença & 202 & 80,5 \\
\hline \multicolumn{3}{|l|}{ Acordo com a instituição em função da amamentação } \\
\hline Sim & 50 & 19,9 \\
\hline Não & 201 & 80,1 \\
\hline \multicolumn{3}{|c|}{$\begin{array}{l}\text { Orientação da instituição sobre a manutenção da amamentação após o } \\
\text { retorno ao trabalho }\end{array}$} \\
\hline Sim & 63 & 25,1 \\
\hline Não & 188 & 74,9 \\
\hline \multicolumn{3}{|c|}{ Conhecimento da existência de banco de leite humano no hospital } \\
\hline Sim & 178 & 70,9 \\
\hline Não & 73 & 29,1 \\
\hline
\end{tabular}

\section{Discussão}

Este estudo tem o mérito de ser o primeiro a ser realizado no Brasil detalhando as características relacionadas ao trabalho da mulher que poderiam interferir no AM após o seu retorno ao trabalho, relacionando-as com a duração dessa prática.

Surpreendentemente, das 22 variáveis relacionadas ao trabalho da mulher, apenas uma mostrou-se associada à continuidade do AM por 12 meses ou mais: o turno de trabalho. Trabalhar somente durante o dia aumentou em $37 \%$ a prevalência de continuidade do AM por 12 meses ou mais. Essa associação já havia sido relatada em outro estudo brasileiro, sugerindo que mulheres que trabalham no período noturno podem ter um distanciamento maior em relação aos cuidados da criança, não conseguindo amamentar em virtude do cansaço decorrente do trabalho noturno ${ }^{12}$.

O presente estudo não encontrou associação entre manutenção do AM após o retorno da mulher ao trabalho e políticas de flexibilização de horário, pausas para amamentar ou extrair o LM, local apropriado para retirada e armazenamento do leite, apoio da chefia e dos colegas de trabalho e creche no local ou próximo ao trabalho. Tais associações foram encontradas em outros estudos ${ }^{6,7,13,14}$. Diferenças importantes entre estes 
Tabela 2. Dados relacionados ao trabalho da mulher e amamentação. Porto Alegre-RS, 2016-2017 (n=251).

\begin{tabular}{|c|c|c|}
\hline Variáveis & $\mathbf{n}$ & $\%$ \\
\hline \multicolumn{3}{|c|}{ Conhecimento da existência de consultoras em lactação no hospital } \\
\hline Sim & 65 & 25,9 \\
\hline Não & 186 & 74,1 \\
\hline \multicolumn{3}{|c|}{$\begin{array}{l}\text { Conhecimento de que tinha direito a } 1 \text { hora a menos de trabalho por dia em } \\
\text { função da amamentação }\end{array}$} \\
\hline $\operatorname{Sim}$ & 226 & 90,0 \\
\hline Não & 25 & 10,0 \\
\hline \multicolumn{3}{|c|}{ Extração de leite da mama durante a jornada de trabalho } \\
\hline Sim & 94 & 37,5 \\
\hline Não & 157 & 62,5 \\
\hline \multicolumn{3}{|l|}{ Utilização da creche da instituição } \\
\hline Sim & 58 & 23,1 \\
\hline Não & 193 & 76,9 \\
\hline \multicolumn{3}{|l|}{ Uso de leite materno extraído da mama na creche } \\
\hline $\operatorname{Sim}$ & 6 & 2,4 \\
\hline Não & 245 & 97,6 \\
\hline \multicolumn{3}{|l|}{ Amamentação na creche } \\
\hline Sim & 34 & 13,5 \\
\hline Não/não frequentava creche & 217 & 86,5 \\
\hline \multicolumn{3}{|c|}{ Sentimento de sobrecarga por conciliar amamentação e trabalho } \\
\hline $\operatorname{Sim}$ & 152 & 60,6 \\
\hline Não & 99 & 39,4 \\
\hline \multicolumn{3}{|c|}{ Percepção de prejuízo ao desempenho no trabalho por causa da amamentação } \\
\hline Sim & 58 & 23,1 \\
\hline Não & 193 & 76,9 \\
\hline
\end{tabular}

${ }^{\star}$ Dados analisados de forma contínua.

Fonte: Elaborado pelas autoras.

estudos e o nosso, tais como características da população estudada e do ambiente de trabalho, bem como duração da licença-maternidade e definição do desfecho, podem explicar, pelo menos em parte, tais diferenças.

$\mathrm{Na}$ população estudada, fatores individuais e outros não vinculados diretamente ao trabalho da mulher associaram-se em maior número com a continuidade do AM por 12 meses ou mais do que fatores relacionados ao trabalho. Os fatores individuais que se mostraram associados com a manutenção do AM após o retorno ao trabalho no presente estudo, tais como idade materna, escolaridade, experiência prévia em amamentação, idade gestacional, uso de outros leites, apoio à amamentação e uso de chupeta têm sido associados com a duração do AM em outros estu$\operatorname{dos}^{11,15-18}$. Esses fatores, portanto, devem ser levados em consideração também na elaboração de estratégias de promoção do AM após retorno ao trabalho.
Um resultado aparentemente paradoxal encontrado no presente estudo foi a associação negativa entre apoio profissional e manutenção da amamentação por mais tempo. É possível que as mulheres que relataram não ter tido apoio profissional tivessem menos problemas na amamentação, necessitando menos desse apoio e, inclusive, procurando menos esses profissionais.

Apesar da importância da informação no manejo da amamentação na fase de retorno ao trabalho ${ }^{17}$, destaca-se, no presente estudo, a desinformação das mulheres. A maioria referiu não ter recebido orientações da instituição sobre o retorno ao trabalho amamentando e não sabia da possibilidade de obter os serviços de consultoras em lactação; algumas desconheciam a existência de BLH na instituição e outras sequer sabiam da possibilidade de redução de jornada de trabalho de 1 hora por dia até a criança completar 1 ano.

A instituição onde foi realizada esta pesquisa, apesar de oferecer várias facilidades que pro- 
Tabela 3. Fatores associados com a continuidade do aleitamento materno por 12 meses ou mais em mulheres trabalhadoras de um hospital geral de grande porte no sul do Brasil - resultado da análise de regressão hierarquizada de Poisson. Porto Alegre-RS, $2016-2017$.

\begin{tabular}{|c|c|c|c|c|c|c|c|}
\hline \multirow[t]{2}{*}{ Variáveis } & \multicolumn{2}{|c|}{$\begin{array}{c}\text { Amostra } \\
(\mathrm{n}=251)\end{array}$} & \multirow{2}{*}{$\begin{array}{c}\text { Prevalência } \\
\mathrm{AM} \geq \mathbf{1 2 m} \\
\%\end{array}$} & \multirow{2}{*}{$\mathrm{RP}_{\mathrm{b}}(\mathrm{IC} 95 \%)$} & \multirow[t]{2}{*}{$\mathbf{p}$} & \multirow{2}{*}{$\mathrm{RP}_{\mathrm{a}}(\mathrm{IC} 95 \%)$} & \multirow[t]{2}{*}{$\mathbf{p}$} \\
\hline & n & $\%$ & & & & & \\
\hline \multicolumn{8}{|l|}{ Bloco Distal } \\
\hline Idade da mãe $(\text { anos) })^{\star}$ - Média (DP) & $35,7(3,7)$ & - & - & $0,97(0,95-0,99)$ & 0,024 & $0,97(0,95-0,99)$ & 0,021 \\
\hline \multicolumn{8}{|l|}{ Mãe com curso superior } \\
\hline Sim & 149 & 59,4 & 56,4 & 1,00 & & 1,00 & \\
\hline Não & 102 & 40,6 & 70,6 & $1,25(1,04-1,51)$ & 0,020 & $1,23(1,02-1,48)$ & 0,033 \\
\hline \multicolumn{8}{|l|}{ Experiência prévia em amamentação } \\
\hline$<12$ meses & 53 & 21,1 & 49,1 & $0,79(0,59-1,07)$ & 0,132 & $0,81(0,60-1,08)$ & 0,152 \\
\hline$\geq 12$ meses & 46 & 18,3 & 78,3 & $1,27(1,04-1,54)$ & 0,019 & $1,32(1,07-1,63)$ & 0,008 \\
\hline Sem experiência & 152 & 60,6 & 61,8 & 1,00 & & 1,00 & \\
\hline \multicolumn{8}{|l|}{ Bloco Intermediário } \\
\hline \multicolumn{8}{|l|}{ Local do pré-natal } \\
\hline Rede pública de saúde & 13 & 5,2 & 84,6 & $1,39(1,08-1,79)$ & 0,011 & $1,03(0,67-1,60)$ & 0,883 \\
\hline Rede privada/Convênio & 238 & 94,8 & 60,9 & 1,00 & & 1,00 & \\
\hline \multicolumn{8}{|l|}{$\begin{array}{l}\text { Intenção quanto à duração da } \\
\text { amamentação }\end{array}$} \\
\hline$\geq 12$ meses & 235 & 93,6 & 64,3 & $2,06(0,99-4,28)$ & 0,054 & $1,79(0,88-3,64)$ & 0,107 \\
\hline$<12$ meses & 16 & 6,4 & 31,3 & 1,00 & & 1,00 & \\
\hline \multicolumn{8}{|l|}{$\begin{array}{l}\text { Intenção de amamentar após o } \\
\text { retorno ao trabalho }\end{array}$} \\
\hline $\operatorname{Sim}$ & 244 & 97,2 & 63,1 & $2,21(0,68-7,16)$ & 0,186 & $1,53(0,49-4,79)$ & 0,470 \\
\hline Não/Talvez & 7 & 2,8 & 28,6 & 1,00 & & 1,00 & \\
\hline \multicolumn{8}{|l|}{ Local do Parto } \\
\hline Rede pública de saúde & 41 & 16,3 & 73,2 & $1,22(0,98-1,51)$ & 0,071 & $1,15(0,88-1,49)$ & 0,305 \\
\hline $\begin{array}{l}\text { Rede privada/Convênio/ } \\
\text { Domicílio }\end{array}$ & 210 & 83,3 & 60,0 & 1,00 & & 1,00 & \\
\hline \multicolumn{8}{|l|}{ Tipo de parto } \\
\hline Vaginal & 45 & 17,9 & 73,3 & $1,23(0,99-1,51)$ & 0,054 & $1,13(0,89-1,42)$ & 0,312 \\
\hline Cesárea & 206 & 82,1 & 59,7 & 1,00 & & 1,00 & \\
\hline $\begin{array}{l}\text { Idade gestacional (semanas) }{ }^{*} \text { - } \\
\text { Média (DP) }\end{array}$ & $38,2(1,7)$ & - & - & $0,95(0,91-0,99)$ & 0,035 & $0,93(0,88-0,97)$ & 0,003 \\
\hline \multicolumn{8}{|l|}{ Sexo da criança } \\
\hline Feminino & 110 & 43,8 & 70,0 & $1,25(1,03-1,51)$ & 0,022 & $1,17(0,97-1,42)$ & 0,106 \\
\hline Masculino & 141 & 56,2 & 56,0 & 1,00 & & 1,00 & \\
\hline \multicolumn{8}{|l|}{ AME na alta da maternidade } \\
\hline Sim & 215 & 85,7 & 64,7 & $1,37(0,96-1,96)$ & 0,087 & $1,39(0,97-1,98)$ & 0,069 \\
\hline Não & 36 & 14,3 & 47,2 & 1,00 & & 1,00 & \\
\hline
\end{tabular}

movem e protegem a amamentação, como por exemplo, ser certificada na Iniciativa Hospital Amigo da Criança, licença-maternidade de 6 meses, disponibilidade de BLH e de profissionais especializados em AM e creche próxima ao local de trabalho, não dispõe de ações direcionadas às mulheres que retornam ao trabalho e querem manter a amamentação. Essa, provavelmente, é a razão de apenas um terço das mulheres terem extraído o seu leite durante a jornada de trabalho, medida muito importante para a manutenção do suprimento de leite. Pode explicar, também, o fato de metade das que extraíam o leite ter o desprezado, mesmo havendo na instituição toda a infraestrutura necessária para a retirada e armazenamento do LM de forma segura, privando, assim, a criança de receber o LM em casa na ausência da mãe.

Um estudo conduzido na Austrália com mulheres profissionais de saúde também mostrou 
Tabela 3. Fatores associados com a continuidade do aleitamento materno por 12 meses ou mais em mulheres trabalhadoras de um hospital geral de grande porte no sul do Brasil - resultado da análise de regressão hierarquizada de Poisson. Porto Alegre-RS, $2016-2017$.

\begin{tabular}{|c|c|c|c|c|c|c|c|}
\hline \multirow[t]{2}{*}{ Variáveis } & \multicolumn{2}{|c|}{$\begin{array}{c}\text { Amostra } \\
(n=251)\end{array}$} & \multirow{2}{*}{$\begin{array}{c}\text { Prevalência } \\
\mathrm{AM} \geq 12 \mathrm{~m}\end{array}$} & \multirow{2}{*}{$\mathrm{RP}_{\mathrm{b}}(\mathrm{IC} 95 \%)$} & \multirow[t]{2}{*}{$\mathbf{p}$} & \multirow{2}{*}{$\mathrm{RP}_{\mathrm{a}}(\mathrm{IC} 95 \%)$} & \multirow[t]{2}{*}{$\mathbf{p}$} \\
\hline & $\mathbf{n}$ & $\%$ & & & & & \\
\hline \multicolumn{8}{|l|}{ Bloco Proximal } \\
\hline $\begin{array}{l}\text { Duração do AME }(\text { dias })^{\star} \text { - Mediana } \\
(\text { P25-P75) }\end{array}$ & $150(90-180)$ & - & - & $\begin{array}{c}1,002 \\
(1,001-1,004)\end{array}$ & 0,007 & $\begin{array}{c}1,001 \\
(1,000-1,003)\end{array}$ & 0,126 \\
\hline \multicolumn{8}{|l|}{$\begin{array}{l}\text { Uso de outro leite quando mãe } \\
\text { retornou ao trabalho }\end{array}$} \\
\hline Sim & 238 & 94,8 & 60,1 & 1,00 & & 1,00 & \\
\hline Não & 13 & 5,2 & 100 & $1,66(1,50-1,85)$ & $<0,001$ & $1,36(1,06-1,74)$ & 0,016 \\
\hline \multicolumn{8}{|l|}{$\begin{array}{l}\text { Apoio do cuidador da criança na } \\
\text { amamentação }\end{array}$} \\
\hline Sim & 203 & 80,9 & 60,1 & $0,85(0,69-1,05)$ & 0,131 & $0,77(0,61-0,97)$ & 0,027 \\
\hline Não/pais são os únicos cuidadores & 48 & 19,1 & 70,8 & 1,00 & & 1,00 & \\
\hline \multicolumn{8}{|l|}{ Apoio profissional na amamentação } \\
\hline Sim & 177 & 70,5 & 58,8 & $0,84(0,69-1,01)$ & 0,069 & $0,78(0,64-0,94)$ & 0,008 \\
\hline Não & 74 & 29,5 & 70,3 & 1,00 & & 1,00 & \\
\hline \multicolumn{8}{|l|}{ Uso de chupeta } \\
\hline Sim & 161 & 64,1 & 56,5 & 1,00 & & 1,00 & \\
\hline Não & 90 & 35,9 & 72,2 & $1,28(1,06-1,54)$ & 0,010 & $1,29(1,07-1,54)$ & 0,006 \\
\hline \multicolumn{8}{|l|}{ Cargo/Função da mãe no hospital } \\
\hline Assistencial & 198 & 78,9 & 63,1 & $1,26(0,89-1,80)$ & 0,195 & $1,33(0,98-1,81)$ & 0,063 \\
\hline Apoio & 19 & 7,6 & 73,7 & $1,47(0,96-2,27)$ & 0,077 & $1,41(0,92-2,17)$ & 0,116 \\
\hline Administrativo & 34 & 13,5 & 50,0 & 1,00 & & 1,00 & \\
\hline \multicolumn{8}{|l|}{ Turno de trabalho da mãe } \\
\hline Diurno & 186 & 74,1 & 66,7 & $1,35(1,04-1,77)$ & 0,026 & $1,37(1,05-1,77)$ & 0,019 \\
\hline Noturno/Ambos & 65 & 25,9 & 49,2 & 1,00 & & 1,00 & \\
\hline \multicolumn{8}{|l|}{$\begin{array}{l}\text { Extração de leite da mama durante a } \\
\text { jornada de trabalho }\end{array}$} \\
\hline $\operatorname{Sim}$ & 94 & 37,5 & 68,1 & $1,16(0,96-1,41)$ & 0,123 & $1,05(0,87-1,26)$ & 0,605 \\
\hline Não & 157 & 62,5 & 58,6 & 1,00 & & 1,00 & \\
\hline \multicolumn{8}{|l|}{$\begin{array}{l}\text { Apoio de colegas de trabalho à } \\
\text { amamentação }\end{array}$} \\
\hline $\operatorname{Sim}$ & 150 & 59,8 & 66,0 & $1,17(0,95-1,44)$ & 0,137 & $1,20(0,99-1,46)$ & 0,070 \\
\hline Não & 101 & 40,2 & 56,4 & 1,00 & & 1,00 & \\
\hline \multicolumn{8}{|l|}{$\begin{array}{l}\text { Orientação da instituição quanto à } \\
\text { continuidade da amamentação após } \\
\text { o retorno ao trabalho }\end{array}$} \\
\hline Sim & 63 & 25,1 & 69,8 & $1,17(0,96-1,43)$ & 0,120 & $1,17(0,96-1,43)$ & 0,124 \\
\hline Não & 188 & 74,9 & 59,6 & 1,00 & & 1,00 & \\
\hline \multicolumn{8}{|l|}{ Utilização da creche da instituição } \\
\hline Sim & 58 & 23,1 & 72,4 & $1,23(1,01-1,49)$ & 0,043 & $0,95(0,68-1,35)$ & 0,785 \\
\hline Não & 193 & 76,9 & 59,3 & 1,00 & & 1,00 & \\
\hline \multicolumn{8}{|l|}{ Amamentação na creche } \\
\hline Sim & 34 & 13,5 & 76,5 & $1,28(1,03-1,58)$ & 0,027 & $1,13(0,80-1,61)$ & 0,493 \\
\hline Não/não frequentava creche & 217 & 86,5 & 59,9 & 1,00 & & 1,00 & \\
\hline \multicolumn{8}{|l|}{$\begin{array}{l}\text { Percepção quanto à dificuldade do } \\
\text { retorno ao trabalho amamentando }\end{array}$} \\
\hline Fácil/Muito fácil & 38 & 15,1 & 76,3 & $1,32(1,05-1,66)$ & 0,019 & $1,14(0,87-1,49)$ & 0,332 \\
\hline Nem fácil, nem difícil & 82 & 32,7 & 62,2 & $1,07(0,86-1,34)$ & 0,541 & $1,02(0,83-1,26)$ & 0,852 \\
\hline Difícil/Muito difícil & 131 & 52,2 & 58,0 & 1,00 & & 1,00 & \\
\hline
\end{tabular}

${ }^{*}$ Dados analisados de forma contínua; $\mathrm{RP}_{\mathrm{b}}=$ Razão de Prevalências Bruta; $\mathrm{RP}=$ =Razão de Prevalências Ajustada; IC95\%=Intervalo de 95\% de confiança; $\mathrm{DP}=$ Desvio Padrão; AM=Aleitamento Materno; AME=Aleitamento Materno Exclusivo; P25-P75=Percentil 25 - Percentil 75.

Fonte: Elaborado pelas autoras. 
desconhecimento delas quanto a aspectos relacionados à amamentação após retorno ao traba$\mathrm{lho}^{19}$. Os autores observaram que muito poucas mulheres conversaram com os gestores sobre amamentação após o retorno ao trabalho e sabiam da pausa para extrair o LM. Mais da metade relatou que provavelmente teria amamentado mais após o retorno ao trabalho se tivesse recebido mais informação e suporte do seu local de trabalho.

No presente estudo, pouco mais da metade das entrevistadas referiu ter recebido apoio de colegas e chefias para continuar a amamentação após o retorno ao trabalho. Apesar do pouco apoio relatado, foi maior do que o observado por Weber et al. ${ }^{7}$ na Austrália, onde apenas $11 \%$ das entrevistadas disseram ter tido apoio da chefia e $13 \%$ dos colegas para manter o AM.É interessante observar também que, na percepção das mulheres do presente estudo, não houve diferença entre o apoio oferecido por colegas homens ou mulheres.

Há relatos de que mães que não conseguem amamentar durante a jornada de trabalho têm mais chances de desmamar ${ }^{20}$ e que manter as crianças perto ou no local de trabalho ajudaria na continuidade do AM após o retorno das mulheres ao trabalho, dando oportunidade para elas oferecerem o peito diretamente ${ }^{6}$. Isto não ocorreu com as mulheres do presente estudo, o que pode ser explicado pelo fato de que muitas crianças que frequentavam a creche não eram amamentadas nesse local. A inadequação do espaço disponível para as mães amamentarem na creche, segundo relatado por elas, pode ter contribuído para a baixa adesão de amamentação nesse local.

É interessante observar que, apesar de não haver um programa institucional de acolhimento às mulheres que retornam ao trabalho após a licença -maternidade e que querem manter a amamentação, a maioria das servidoras da instituição onde o estudo foi conduzido relatou estar satisfeita com o apoio recebido, o que pode ser um reflexo da satisfação geral da trabalhadora com a instituição.

Outro achado interessante deste estudo foi a maior duração do AME entre as mulheres estudadas quando comparadas com a população do município de Porto Alegre (150 dias vs. 52 dias $^{8}$ ).
Uma das hipóteses para esse achado é o fato de a maioria das mulheres de nosso estudo ser profissional de um hospital que valoriza a amamentação, haja vista ser certificado pela Iniciativa Hospital Amigo da Criança. A licença-maternidade de 6 meses, somada ao fato de que as mulheres que desmamaram antes de retornarem ao trabalho não fizeram parte do estudo, pode ter contribuído para esse resultado.

Apesar da relevância dos achados deste estudo, é importante apontar algumas limitações. Destaca-se o fato de ter sido realizado em um único local e com uma população constituída na sua maioria por profissionais de saúde. Além disso, as mulheres deste estudo têm alguns privilégios que a maioria das trabalhadoras no Brasil não possui, como licença-maternidade de 6 meses, alta escolaridade, disponibilidade de um BLH e creche próximo ao local de trabalho. Por isso, são necessárias pesquisas futuras em locais com estrutura e organização diferentes para as mulheres que amamentam e com outros perfis de população para um melhor entendimento dos fatores no ambiente de trabalho que facilitam ou dificultam a continuidade do AM após o retorno da mulher ao trabalho.

Concluindo, este estudo mostrou que, entre as servidoras da instituição pesquisada, os fatores ligados diretamente ao trabalho da mulher que poderiam interferir negativamente na manutenção do AM após o retorno da mulher ao trabalho não se associaram à continuidade do AM por 12 meses ou mais. Isto pode ser explicado, pelo menos em parte, pelos benefícios oferecidos pela instituição, sobretudo a licença-maternidade de 6 meses. Apesar disso, é provável que um programa de acolhimento das mulheres que querem manter a amamentação após o retorno ao trabalho poderia mudar esse cenário e aumentar as prevalências de amamentação continuada aos 12 meses, além de tornar mais fácil e prazerosa a experiência de conciliar trabalho materno e amamentação. Os fatores associados à continuidade do AM encontrados neste estudo, inclusive os não relacionados ao local de trabalho, devem ser levados em consideração na elaboração de estratégias de promoção do AM após retorno ao trabalho. 


\section{Colaboradores}

MS Mendes contribuiu em todas as etapas, desde a coleta dos dados, tabulação, análise e redação. M Schorn contribuiu nas etapas de coleta dos dados, tabulação e análise. LC Espírito Santo contribuiu na orientação para: a coleta dos dados, análise, redação e concepção do estudo. LD Oliveira contribuiu na orientação para: coleta dos dados, análise, redação e concepção do estudo. ERJ Giugliani contribuiu na orientação para: coleta dos dados, análise, redação e concepção do estudo.

\section{Referências}

1. Victora CG, Bahl R, Barros AJD, França GVA, Horton S, Krasevec J, Murch S, Sankar MJ, Walker N, Rollins NC, Lancet Breastfeeding Series Group. Breastfeeding in the 21st century: epidemiology, mechanisms, and lifelong effect. Lancet 2016; 387(10017):475-490.

2. Grummer-Strawn LM, Rollins N. Summarising the health effects of breastfeeding. Acta Paediatr 2015; 104:1-2.

3. Stuebe A. The risks of not breastfeeding for mothers and infants. Rev Obstet Gynecol 2009; 2(4):222-231.

4. Warkentin S, Taddei JAAC, Viana K J, Colugnati FAB. Exclusive breastfeeding duration and determinants among Brazilian children under two years of age. Rev Nutr 2013; 26(3):259-269.

5. Rea MF, Venâncio SI, Batista LE, Santos RG, Greiner T. Possibilidades e limitações da amamentação entre mulheres trabalhadoras formais. Rev Saude Publica 1997; 31(2):149-156.

6. Fein SB, Mandal B, Roe BE. Success of strategies for combining employment and breastfeeding. Pediatrics 2008; 122(Supl. 2):S56-S62.

7. Weber D, Janson A, Nolan M, Wen L, Rissel C. Female employees' perceptions of organisational support for breastfeeding at work: findings from an Australian health service workplace. Int Breastfeed J 2011; 6(1):19.

8. Brasil. Ministério da Saúde (MS). II Pesquisa de Prevalência de Aleitamento Materno nas Capitais Brasileiras e Distrito Federal. Brasília: MS; 2009.

9. Brasil. Ministério da Saúde (MS). Pesquisa Nacional de Demografia e Saúde da Criança e Mulher - PNDS 2006. Brasília: MS; 2009.

10. Victora CG, Huttly SR, Fuchs SC, Olinto MT. The role of conceptual frameworks in epidemiological analysis: a hierarchical approach. Int J Epidemiol 1997; 26(1):224-227.

11. Boccolini CS, Carvalho ML, Oliveira MI. Factors associated with exclusive breastfeeding in the first six months of life in Brazil: a systematic review. Rev Saude Publica 2015; 49:91.

12. Morais AMB, Machado MMT, Aquino PS, Almeida MI. Vivência da amamentação por trabalhadoras de uma indústria têxtil do Estado do Ceará, Brasil. Rev Bras Enferm 2011; 64(1):66-71.
13. Dinour LM, Szaro JM. Employer-based programs to support breastfeeding among working mothers: A systematic review. Breastfeed Med 2017; 12(3): 131141.

14. Marinelli KA, Moren K, Taylor JS, The Academy of Breastfeeding Medicine.Breastfeeding support for mothers in workplace employment or educational settings: summary statement. Breastfeed Med 2013; 8(1):137-142.

15. Muelbert M. Fatores Associados com a Manutenção do Aleitamento Materno por 6, 12 e 24 meses em uma coorte de mães adolescentes [dissertação]. Porto Alegre: Universidade Federal do Rio Grande do Sul; 2017.

16. Boccolini CS, Carvalho ML, Oliveira MIC, Boccolini PMM. Breastfeeding can prevent hospitalization for pneumonia among children under 1 year old. J Pedia$\operatorname{tr}$ (Rio J) 2011; 87(5):399-404.

17. Brasileiro AA, Ambrosano GMB, Marba STM, Possobon RF. A amamentação entre filhos de mulheres trabalhadoras. Rev Saude Publica 2012; 46(4):642-648.

18. Martins EJ, Giugliani ERJ. Which women breastfeed for 2 years or more? J Pediatr (Rio J) 2012; 88(1):6773.

19. Xiang N, Zadoroznyj M, Tomaszewski W, Martin B. Timing of return to work and breastfeeding in Australia. Pediatrics 2016; 137(6):e20153883.

20. Brasileiro AA, Possobon RF, Carrascoza KC, Ambrosano GMB, Moraes ABA. Impacto do incentivo ao aleitamento materno entre trabalhadoras formais. Cad Saude Publica 2010; 26(9):1705-1713.

Artigo apresentado em 04/02/2020

Aprovado em 19/10/2020

Versão final apresentada em 21/10/2020

Editores-chefes: Romeu Gomes, Antônio Augusto Moura da Silva 